\title{
Neues zu Diagnostik und Therapie des idiopathischen Parkinson-Syndroms
}

Josefine Waldthaler, Lars Timmermann

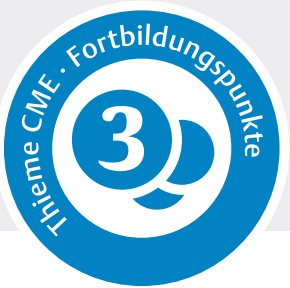

In Deutschland leben mindestens 300000 Menschen mit einem idiopathischen Parkinson-Syndrom. Aufgrund des demografischen Wandels ist mit einer stetig steigenden Zahl Betroffener zu rechnen. In diesem Beitrag werden die evidenzbasierte Diagnostik und Therapie des idiopathischen Parkinson-Syndroms dargestellt. Insbesondere soll ein Überblick über die neuen Erkenntnisse und Entwicklungen der letzten Jahre gegeben werden.

\section{Epidemiologie}

Das idiopathische Parkinson-Syndrom (IPS) ist mit einer Inzidenz von 100-200 Neuerkrankungen pro 100000 Einwohnern pro Jahr in Deutschland nach Morbus Alzheimer die zweithäufigste neurodegenerative Erkrankung.

Die Prävalenz der Parkinson-Krankheit nimmt mit dem Alter zu: Fast die Hälfte der Betroffenen erkrankt zwischen dem 50. und dem 60. Lebensjahr, weitere $20 \%$ in noch höherem Alter. Mit dem demografischen Wandel und der steigenden Lebenserwartung ist somit mit einer Zunahme der Zahl Betroffener zu rechnen.

\section{Ätiologie}

Was der Auslöser für den Verlust dopaminerger Neuronen beim IPS ist, bleibt weiterhin unklar. Es wird ein Zusammenspiel von Umweltfaktoren, Lebensstil und genetischer Prädisposition angenommen. Bekannte Risikofaktoren sind:

- Alter,

- männliches Geschlecht,

- Exposition gegenüber Mangan oder Pestiziden (z. B. Rotenon, Paraquat). Genuss von Nikotin und Koffein scheinen das Risiko zu vermindern, an einem IPS zu erkranken.

Die große Mehrheit der Fälle ist sporadisch. Monogen erbliche Formen machen ca. 5-10\% aus. Daneben kommen familiäre Häufungen vor, ohne dass eine bekannte genetische Ursache identifiziert werden kann.

$\begin{array}{ll}\text { ABKÜRZUNGEN } & \\ \text { CBS } & \text { kortikobasales Syndrom } \\ \text { COMT } & \text { Catechol-O- } \\ & \text { Methyltransferase }\end{array}$

\begin{tabular}{|c|c|}
\hline DBS & Deep Brain Stimulation \\
\hline DGN & $\begin{array}{l}\text { Deutsche Gesellschaft für } \\
\text { Neurologie }\end{array}$ \\
\hline FDG-PET & $\begin{array}{l}{ }^{18} \text { F-Fluordesoxyglukose-Posit- } \\
\text { ronenemissionstomografie }\end{array}$ \\
\hline FP-CIT-Spect & 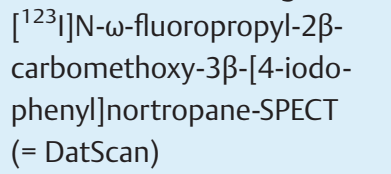 \\
\hline GPi & Globus pallidus internus \\
\hline IPS & $\begin{array}{l}\text { idiopathisches Parkinson- } \\
\text { Syndrom }\end{array}$ \\
\hline LCIG & $\begin{array}{l}\text { L-Dopa-Carbidopa Intesti- } \\
\text { nal Gel }\end{array}$ \\
\hline LED & L-Dopa-Äquivalenzdosis \\
\hline MAO-B & Monoaminoxidase B \\
\hline $\mathrm{MCl}$ & Mild cognitive Impairment \\
\hline MDS & Movement Disorders Society \\
\hline MIBG-Szintigrafie & $\begin{array}{l}\text { Metaiodbenzylguanidin- } \\
\text { Szintigrafie }\end{array}$ \\
\hline MoCA & $\begin{array}{l}\text { Montreal Cognitive } \\
\text { Assessment }\end{array}$ \\
\hline MSA & Multisystematrophie \\
\hline NMDA & N-Methyl-D-Aspartat \\
\hline PARC & $\begin{array}{l}\text { Parkinson associated Risk } \\
\text { Complex }\end{array}$ \\
\hline PARK & $\begin{array}{l}\text { Parkinson's disease associa- } \\
\text { ted genes }\end{array}$ \\
\hline PD & Parkinson's Disease \\
\hline PEG & $\begin{array}{l}\text { perkutane endoskopische } \\
\text { Gastrostomie }\end{array}$ \\
\hline PEJ & $\begin{array}{l}\text { perkutane endoskopische } \\
\text { Jejunostomie }\end{array}$ \\
\hline PSP & $\begin{array}{l}\text { Progressive supranuclear } \\
\text { Palsy }\end{array}$ \\
\hline RCT & Randomised clinical Trial \\
\hline
\end{tabular}




$\begin{array}{ll}\text { RCT } & \text { Randomized controlled trial } \\ \text { REM } & \text { Rapid Eye Movement } \\ \text { SSRI } & \begin{array}{l}\text { selektiver Serotonin-Reup- } \\ \text { take-Inhibitor }\end{array} \\ & \text { subthalamischer Nukleus } \\ \text { STN } & \text { tiefe Hirnstimulation } \\ \text { THS } & \text { Unified Parkinson's Disease } \\ \text { UPDRS } & \text { Rating Scale } \\ & \text { ventraler intermedialer } \\ \text { Vim } & \text { Nukleus } \\ & \text { zentrales Nervensystem }\end{array}$

\section{Verlauf}

Die Erkrankung verläuft insgesamt sehr individuell. Allgemein sind früherer Beginn, Tremordominanz und weibliches Geschlecht positive prognostische Faktoren für einen milderen Langzeitverlauf.

\section{Diagnose}

\section{Diagnosekriterien}

\section{FALLBEISPIEL \\ Erstdiagnose}

Ein 58-jähriger Mann leidet seit ca. einem halben Jahr an Steifigkeit und Schmerzen im rechten Arm und einer Störung der Feinmotorik der rechten Hand. Seine Ehefrau habe sich beschwert, dass er beim Spazierengehen so langsam geworden sei. Außerdem schwinge sein rechter Arm nicht mehr mit, und die Hand zittere beim Gehen.

Vor einigen Jahren habe er eine Depression gehabt, sonst sei er nie ernsthaft krank gewesen. Ein Schädel-MRT sei unauffällig verlaufen. Auswärts wurde bereits ein Parkinson-Syndrom diagnostiziert und retardiertes Pramipexol verschrieben, das er aber noch nicht eingenommen habe.

In der klinischen Untersuchung zeigt sich eine einseitige Bradykinese mit leichtem Extremitätenrigor und einem Ruhetremor rechts. Zusammenfassend besteht bei dem Patienten somit ein klinisch wahrscheinliches rechtsbetontes idiopathisches Parkinson-Syndrom vom Äquivalenztyp im Stadium 1 nach Hoehn und Yahr. Er habe eine medikamentöse Therapie bisher hinauszögern wollen, um Tabletten einzusparen. Andererseits sei er sehr aktiv und fühle sich inzwischen durch die herabgesetzte Beweglichkeit bei der Arbeit und beim Sport deutlich eingeschränkt. Deshalb wird vereinbart, die Therapie nun zu beginnen - auch um das Ansprechen der Symptome zu überprüfen.

\section{Merke}

Im klinischen Alltag fußt die Diagnose eines IPS weiterhin auf der gründlichen Anamnese, der vollständigen neurologischen Untersuchung und dem Ansprechen der Symptomatik auf L-Dopa.

Die Bradykinese stellt hierbei das entscheidende Symptom dar: Hiermit wird die Verlangsamung der Initiierung und Durchführung willkürlicher Bewegungen sowie eine Abnahme der Amplitude bei wiederholten Bewegungen beschrieben. Die Begriffe Bradykinese, Akinese und Hypokinese werden meist synonym verwendet.

Die UK-Brain-Bank-Kriterien von 1992 [3] galten über Jahrzehnte als der Goldstandard für die klinische Diagnose des IPS. Sie stellen die einzigen Kriterien dar, die mehrfach gegen die definitive neuropathologische Diagnose post mortem validiert wurden. Studien haben allerdings inzwischen gezeigt, dass erfahrene Untersucher die Diagnose eines IPS mit größerer Genauigkeit stellen, als es durch die strenge Anwendung der UK-Brain-BankKriterien allein möglich ist.

2015 hat die Movement Disorders Society (MDS) neue Diagnosekriterien vorgestellt, die die entscheidende Rolle eines spezialisierten Neurologen besonders betonen. Neben neu definierten Ausschlusskriterien und Red Flags wurde auch die Symptomentwicklung im zeitlichen Verlauf stärker berücksichtigt als bei vorangegangen Klassifikationen. Außerdem hat Zusatzdiagnostik Beachtung gefunden, für die ein klarer Nachweis ihrer hohen Spezifität und Sensitivität besteht. Die neuen Kriterien sollen somit durch stärke Standardisierung den diagnostischen Prozess reproduzierbar machen [1].

Die aktuelle Version der Leitlinie der Deutschen Gesellschaft für Neurologie (DGN) von 2016 bezieht sich auf die langjährig anerkannten UK-Brain-Bank-Kriterien [2], [3]. In $>$ Tab. 1 sind daher die Kriterien vergleichend dargestellt.

Die MDS-Diagnosekriterien unterscheiden ein klinisch etabliertes IPS von einem klinisch wahrscheinlichen IPS. Für beide ist das Fehlen von Ausschlusskriterien Voraussetzung.

Für die Diagnose des etablierten IPS müssen zwei unterstützende Merkmale und keine Red Flags vorliegen. Ein klinisch wahrscheinliches IPS ist definiert durch das sich ausgleichende Vorliegen von unterstützenden Merkmalen und bis zu zwei Red Flags, d. h. wenn ein Red-FlagKriterium vorhanden ist, muss mindestens auch ein unterstützendes Merkmal vorliegen.

Zum Staging des IPS wird weiterhin die Einteilung nach Hoehn und Yahr verwendet. Die motorische Symptomschwere wird in der Unified Parkinson's Disease Rating 


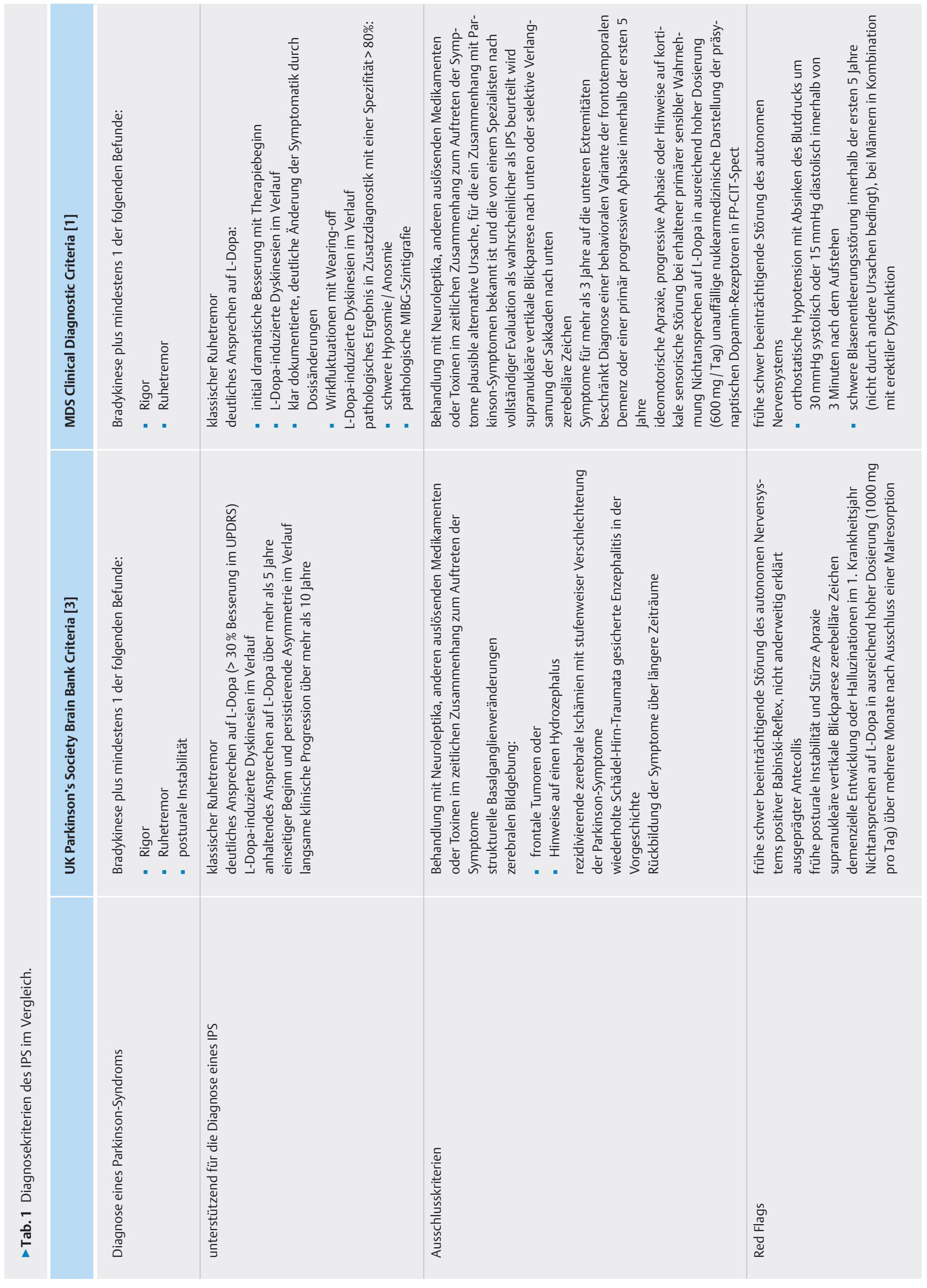




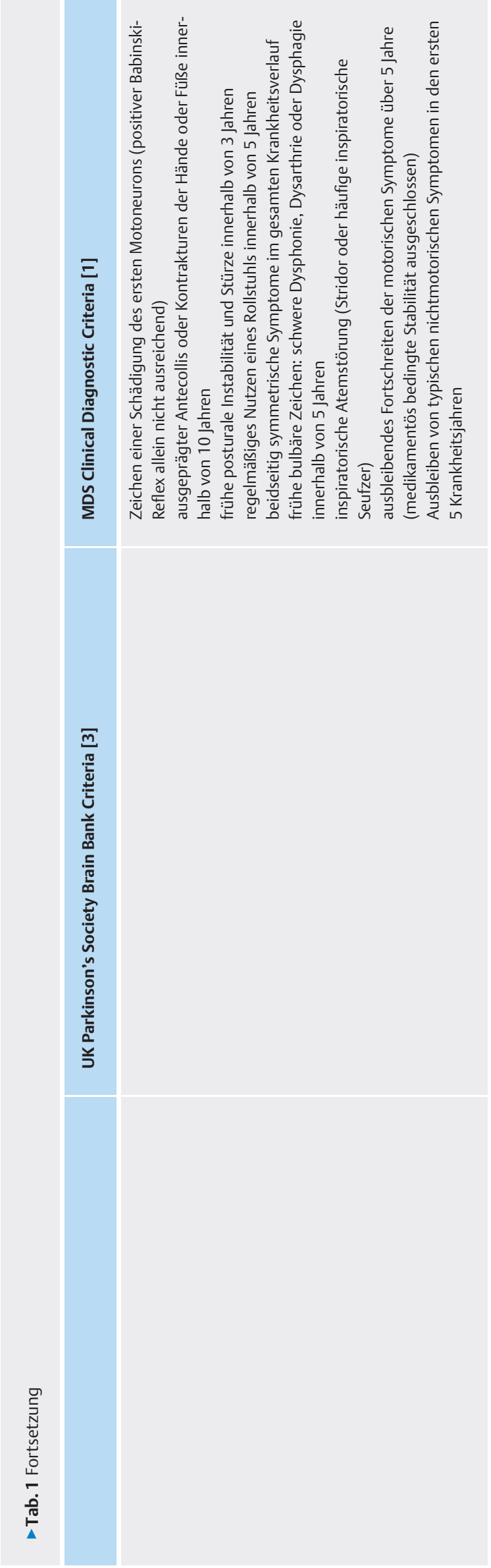


Scale (UPDRS) erfasst, die in den letzten Jahren zunehmend von einer neuen Fassung, der MDS-UPDRS, abgelöst wurde. Wichtig zu beachten ist, dass hier zusätzliche Items enthalten sind, sodass die Summenwerte beider Scores nicht direkt vergleichbar sind.

\section{Ansprechen auf L-Dopa}

Das Ansprechen auf L-Dopa kann mit einem standardisierten Dopa-Test oder durch probatorische, chronische Behandlung überprüft werden.

\section{Merke}

Gerade bei De-novo-Patienten ist die Aussagekraft des Dopa-Tests zur Differenzierung eines IPS von atypischen Parkinson-Syndromen geringer als die der chronischen Behandlung mit L-Dopa.

Daher sollte bei klinischem Vorliegen eines ParkinsonSyndroms immer ein ausreichend hoch dosierter Therapieversuch mit L-Dopa (wenn nötig schrittweise bis $1000 \mathrm{mg}$ pro Tag) über einen ausreichend langen Zeitraum von einigen Wochen erfolgen. In bestimmten Situationen kann der Dopa-Test dennoch zum schnelleren und objektiveren Abschätzen des Effektes von LDopa durchaus sinnvoll sein.

Der Apomorphin-Test ist nach aktueller Studienlage dem Dopa-Test nicht überlegen. Apomorphin, das nur s. c. verabreicht werden kann, hat gleichzeitig das höhere Nebenwirkungspotenzial für Übelkeit, Erbrechen und relevanten Blutdruckabfall. Deshalb sollte dem DopaTest der Vorzug gegeben werden, wenn keine Hinweise auf eine Malresorption vorliegen.

Zur Überprüfung des Therapieerfolgs und damit auch der Diagnose sollte die erste Follow-up-Untersuchung nach 3 Monaten erfolgen. Anschließend sind regelmäBige Wiedervorstellungen nach Bedarf, aber mindestens in einem Intervall von einem Jahr anzuraten.

\section{Bildgebung}

Ein MRT des Schädels ist notwendig, um strukturelle Ursachen auszuschließen.Während beim IPS ein altersentsprechend unauffälliges MRT zu erwarten ist, finden sich bei atypischen Parkinson-Syndromen mitunter verschiedene bildgebende Hinweise, z. B., um nur einige zu nennen:

- eine reduzierte Mittelhirn-Pons-Ratio und das „Kolibri“Zeichen bei der Progressive supranuclear Palsy (PSP),

- die einseitige kortikale Atrophie beim kortikobasalen Syndrom (CBS) oder

- das „Hot-Cross-Bun“ bei der Multisystematrophie (MSA).

Viele dieser Zeichen, die meistens erst im Verlauf der Erkrankungen deutlich erkennbar werden, zeichnet eine relativ hohe Spezifität aus. Die Sensitivität ist allerdings insgesamt gering.

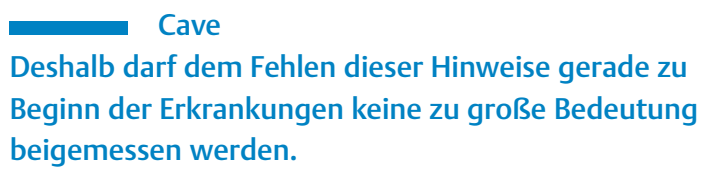

Die Verbesserung der MRT-Scanner in den letzten Jahren hat eine direkte Darstellung einer krankheitsspezifischen Hyperintensität der dorsalen Substantia nigra bei IPS ermöglicht. Die Auffälligkeiten in den sogenannten Nigrosomen in $\mathrm{T} 2{ }^{*}$-Sequenzen bieten eine hohe Sensitivität und Spezifität zur Unterscheidung von Parkinson-Patienten von Gesunden, aber bisher nicht in der Abgrenzung der verschiedenen Parkinson-Syndrome untereinander. Aufgrund der eingeschränkten Verfügbarkeit von 3- oder 7-Tesla-Geräten in der klinischen Anwendung hat die Nigrosomen-Darstellung momentan allerdings nur selten Relevanz in der klinischen Diagnostik [2].

\section{Merke \\ Nuklearmedizinische Diagnostik bleibt klinisch unein- deutigen, begründeten Fällen unter Abwägung von Nutzen und Risiko (Strahlenbelastung!) vorbehalten.}

Das 123I-FP-CIT-SPECT oder DatScan ${ }^{\mathrm{TM}}$ stellt den postsynaptischen Dopamintransporter DaT dar. Eine Minderanreicherung des Tracers in den Basalganglien stützt klar die Diagnose eines neurodegenerativen Parkinson-Syndroms, z. B. in Abgrenzung zum essenziellen Tremor oder einem Neuroleptika-induzierten Parkinsonoid.

Zur Differenzierung des IPS von atypischen ParkinsonSyndromen wurde in den letzten Jahren das IBZM-Spect zunehmend vom 18-Fluoro-Deoxyglucose-Positronenemissionstomogramm (FDG-PET) des Gehirns abgelöst, das eine höhere Sensitivität und Spezifität aufweist und nicht durch dopaminerge Medikation verfälscht wird. Die verschiedenen Parkinson-Syndrome unterscheiden sind hier in ihrem typischen Verteilungsmuster der herabgesetzten Glukoseverstoffwechselung [5].

Zur Unterscheidung einer Multisystematrophie (MSA) von einem idiopathischen Parkinson-Syndrom kann die MIBG-Szintigrafie des Myokards hilfreich sein.

\section{Genetische Testung}

In insgesamt ca. 5-10\% aller IPS-Fälle findet sich eine klar monogen erbliche Form mit Nachweis einer auslösenden Mutation im einem der bereits identifizierten Gene [6]. Nicht alle der 20 ursprünglich in als „PARK“ klassifizierten Genloci haben sich in wiederholten Analysen bestätigt. Einige führen zu einem anderen Phänotyp, der sich deutlich von einem IPS unterscheidet. Die klinisch relevanten und bestätigten genetischen 
\ab. 2 Bestätigte monogene Ursachen eines IPS-Phänotyps.

\begin{tabular}{|c|c|c|c|c|}
\hline & Gen & Häufigkeit & $\begin{array}{l}\text { Phänotyp, } \\
\text { Erkrankungsalter }\end{array}$ & Besonderheiten \\
\hline \multicolumn{5}{|c|}{ autosomal-dominant } \\
\hline $\begin{array}{l}\text { PARK } 1 \\
\text { PARK } 4\end{array}$ & SNCA & & $\begin{array}{l}\text { Early Onset / klassi- } \\
\text { sches IPS (je nach } \\
\text { Mutation Alter 30-60 } \\
\text { Jahre) }\end{array}$ & $\begin{array}{l}\text { Punktmutationen und } \\
\text { Duplikationen; zusätz- } \\
\text { lich Risikovarianten, } \\
\text { bei Duplikationen häu- } \\
\text { fig Demenz }\end{array}$ \\
\hline PARK 8 & LRRK2 & $\begin{array}{l}\text { ca. } 5 \% \text { der familiären } \\
\text { und } 3 \% \text { der sporadi- } \\
\text { schen IPS-Fälle }\end{array}$ & klassisches IPS & $\begin{array}{l}\text { sowohl krankheitsver- } \\
\text { ursachende Mutatio- } \\
\text { nen als auch } \\
\text { Risikovarianten, } \\
\text { inkomplette Penetranz }\end{array}$ \\
\hline PARK 17 & VPS35 & einzelne Familien & klassisches IPS & \\
\hline \multicolumn{5}{|c|}{ autosomal-rezessiv } \\
\hline PARK 2 & Parkin & $\begin{array}{l}\text { bis zu } 18 \% \text { der Early- } \\
\text { Onset-IPS-Fälle }\end{array}$ & $\begin{array}{l}\text { Early-Onset-IPS (20-40 } \\
\text { Jahre) }\end{array}$ & $\begin{array}{l}\text { langsam progredient, } \\
\text { sehr gutes Ansprechen } \\
\text { auf L-Dopa; oft } \\
\text { Fußdystonie }\end{array}$ \\
\hline PARK 6 & PINK-1 & $\begin{array}{l}4 \% \text { der Early-Onset- } \\
\text { IPS-Fälle }\end{array}$ & Early-Onset-IPS & $\begin{array}{l}\text { ähnlicher Phänotyp } \\
\text { wie PARK2 }\end{array}$ \\
\hline PARK 7 & DJ-1 & $\begin{array}{l}\text { ca. } 1 \% \text { der Early- } \\
\text { Onset-IPS-Fälle }\end{array}$ & Early-Onset-IPS & $\begin{array}{l}\text { ähnlicher Phänotyp } \\
\text { wie PARK } 2 \text { und } 6 \text {, } \\
\text { eventuell mehr psy- } \\
\text { chiatrische Symptome }\end{array}$ \\
\hline
\end{tabular}

Zusätzlich werden immer mehr genetische Varianten in bestimmten Suszeptibilitätsgenen (besonders hervorzuheben GBA, LRKK2, SNCA, MAPT) durch genomweite Assoziationsstudien identifiziert, die das Erkrankungsrisiko des IPS modulieren. Ihr Vorkommen in unterschiedlichen Populationen wie auch der Einfluss verschiedener Varianten auf das Erkrankungsrisiko sind sehr variabel.

Ursachen eines IPS sind in $>$ Tab. 2 dargestellt. Die autosomal-rezessiv vererbten Varianten führen zu einer sehr frühen Erstmanifestation, während der Krankheitsbeginn bei den autosomal-dominant vererbten Formen variabel und später liegt.

\section{Merke}

Zusammenfassend ist daher bei Patienten mit einer Krankheitsmanifestation vor dem 45. Lebensjahr oder bei mindestens zwei ebenfalls betroffenen Verwandten ersten Grades eine humangenetische Beratung und Testung sinnvoll und gerechtfertigt.

\section{Nichtmotorische Symptome}

In den letzten Jahren rücken die nichtmotorischen Symptome immer weiter in den Fokus der wissenschaftlichen und klinischen Aufmerksamkeit, die zum Teil Jahre vor der klinischen Manifestation der motorischen Symptome auftreten. So zeigen Individuen mit dem sogenannten Parkinson associated Risk Complex (PARC) noch keine motorischen Kardinalsymptome, weisen aber eine Kombination der typischen nichtmotorischen Beschwerden - insbesondere Störung des REM-Schlafverhaltens, Riechstörung, Depression, Obstipation - auf, die mit einem deutlich erhöhten Risiko für das Auftreten eines IPS assoziiert sind.

Die Diagnose eines PARC hat aktuell zwar keine bzw. eine rein symptomatische Therapiekonsequenz der einzelnen Beschwerden. Die Identifizierung dieser Patienten verspricht allerdings den potenziell größten therapeutischen Nutzen zukünftiger krankheitsmodifizierender Therapien. Hierfür hat die MDS ebenfalls bereits Diagnosekriterien vorgelegt [7].

Die Ausprägung der verschiedenen nichtmotorischen Beschwerden ist von Patient zu Patient sehr unterschiedlich.

\footnotetext{
Cave

Das Vorliegen kognitiver Defizite, einer Störung des REM-Schlafverhaltens und einer orthostatischen Dysregulation sind ungünstige Faktoren für die Langzeitprognose [8].
} 


\section{Therapieoptionen des IPS}

\section{Medikamentöse Primärtherapie}

Die medikamentöse Primärtherapie der motorischen Symptome des IPS fasst $\triangleright$ Tab. 3 zusammen [9].

Die Symptome und der Verlauf des IPS sind sehr individuell. Daher sollten auch die persönlichen Anforderungen des Patienten an seine Medikation - unter anderem Berufstätigkeit, Freizeitaktivitäten, soziale Aspekte, Lebensqualität, Pflegebedürftigkeit und Nebenerkrankungen - eine wichtige Rolle bei der Auswahl der optimalen Therapie spielen.

\section{Merke}

Es bleibt hervorzuheben, dass für keines der zugelassenen Medikamente eine krankheitsmodifizierende Wirkung ausreichend belegt ist.
Der als Ergebnis der ADAGIO-Studie vermutete neuroprotektive Effekt hat sich in der Folge nicht bestätigt. Aktuell existiert keine ausreichende Evidenz für einen krankheitsmodifizierenden Effekt der MAO-B-Hemmer.

\section{FALLBEISPIEL \\ Impulskontrollstörung}

Der gleiche Patient stellt sich 3 Monate später erneut vor und berichtet, dass er sich unter Einnahme von Pramipexol sehr gut fühle. An manchen Tagen vergesse er die Erkrankung völlig.

Auch in der klinischen Untersuchung zeigt sich eine deutliche Besserung des UPDRS.

Erst zum Ende des Besuchs hin gibt der Patient an, doch noch eine Auffälligkeit festgestellt zu haben, die ihm etwas unangenehm sei. Seit einigen

- Tab. 3 Medikamentöse Therapie der motorischen Symptome des IPS [9].

\begin{tabular}{|c|c|c|c|}
\hline Substanzklasse & $\begin{array}{l}\text { in Deutschland zugelassene } \\
\text { Substanzen }\end{array}$ & $\begin{array}{l}\text { wichtige Nebenwirkungen } \\
\text { (Auswahl) }\end{array}$ & Bemerkungen \\
\hline L-Dopa + Carboxylasehemmer & $\begin{array}{l}\text { L-Dopa + Carbidopa L-Dopa + } \\
\text { Benserazid auch als retar- } \\
\text { dierte und schnell wirksame } \\
\text { Präparate }\end{array}$ & $\begin{array}{l}\text { Übelkeit Blutdruckabfall, } \\
\text { Schwindel Dyskinesien im } \\
\text { Verlauf }\end{array}$ & $\begin{array}{l}\text { als Monotherapie und in } \\
\text { Kombination }\end{array}$ \\
\hline $\begin{array}{l}\text { Non-Ergot- } \\
\text { Dopaminagonisten* }\end{array}$ & $\begin{array}{l}\text { Rotigotin (transdermal) Pra- } \\
\text { mipexol Pramipexol retard } \\
\text { Pirebedil Ropinirol Ropinirol } \\
\text { retard Apomorphin (s. c.) }\end{array}$ & $\begin{array}{l}\text { Übelkeit, Blutdruckabfall, } \\
\text { Schwindel Tagesmüdigkeit, } \\
\text { Einschlafattacken - Cave: Die } \\
\text { Fahrtauglichkeit kann einge- } \\
\text { schränkt sein! Impulskontroll- } \\
\text { störung - Cave! Verwirrtheit, } \\
\text { Halluzinationen Rotigotin und } \\
\text { Apomorphin: } \\
\text { Hautveränderungen }\end{array}$ & $\begin{array}{l}\text { als Monotherapie des frühen } \\
\text { IPS (bei jungen Patienten) } \\
\text { und in Kombination }\end{array}$ \\
\hline MAO-B-Hemmer & Rasagilin Selegilin & $\begin{array}{l}\text { Übelkeit, Schwindel Müdigkeit } \\
\text { Cave: Wechselwirkungen mit } \\
\text { anderen MAO-Hemmern, } \\
\text { Johanniskraut, SSRI, Sympa- } \\
\text { thomimetika u. a. }\end{array}$ & $\begin{array}{l}\text { Monotherapie des frühen IPS } \\
\text { und als Add-on bei } \\
\text { Fluktuationen }\end{array}$ \\
\hline $\begin{array}{l}\text { - zusätzlich Dopamin- und } \\
\text { Glutamat-Modulator }\end{array}$ & Safinamid & - & $\begin{array}{l}\text { nur als Add-on bei Fluktuatio- } \\
\text { nen und Dyskinesien }\end{array}$ \\
\hline COMT-Hemmer & Entacapon Tolcapon Opicapon & $\begin{array}{l}\text { Übelkeit, Schwindel, Schwit- } \\
\text { zen Diarrhö Dyskinesien } \\
\text { harmlose Gelbfärbung des } \\
\text { Urins Tolcapon: Leberfunk- } \\
\text { tionsstörung - Cave: regel- } \\
\text { mäßige Kontrollen der } \\
\text { Leberfunktion erforderlich! }\end{array}$ & $\begin{array}{l}\text { nur als Add-on zu L-Dopa bei } \\
\text { Fluktuationen }\end{array}$ \\
\hline NMDA-Antagonisten & Amantadin & $\begin{array}{l}\text { Schwindel, Übelkeit anticholi- } \\
\text { nerg Nervosität, Unruhe Ver- } \\
\text { wirrtheit, Halluzinationen QT- } \\
\text { Zeit-Verlängerung }\end{array}$ & $\begin{array}{l}\text { vorwiegend als Add-on bei } \\
\text { Dyskinesien oder } \\
\text { Gangstörung }\end{array}$ \\
\hline
\end{tabular}

* Ergot-Dopaminagonisten (nicht aufgeführt) wie Bromocriptin und Cabergolin dürfen aufgrund ihres sehr ungünstigen Nebenwirkungsprofils in Hinblick auf Fibrosen nur noch in Ausnahmefällen unter strenger Beachtung der Fachinformation eingesetzt werden. 
Monaten gehe er nach der Arbeit gelegentlich ins Casino und setze dort mitunter Geldbeträge, die ihm im Nachhinein manchmal zu hoch vorkämen. Dies sei sonst gar nicht seine Art, und mit seiner Frau sei es deshalb schon mehrfach zu Streitereien gekommen. Dann sei er auch viel aufbrausender als früher. Auf Nachfrage berichtete er, an Gewicht zugenommen zu haben, weil er oft spätabends den Kühlschrank „plündere“.

Die Symptome sind Folge einer Impulskontrollstörung und damit der Therapie mit dem Dopaminagonisten. Dem Patienten wird dringend geraten, die Therapie umzustellen. Nach einem Wechsel auf Ropinirol sistieren die Symptome fast vollständig. Nur bei Süßigkeiten könne er sich ab und zu nicht beherrschen ...

\section{PRAXIS}

\section{Impulskontrollstörung}

Impulskontrollstörungen treten bei ca. 15\% der Patienten unter einer Therapie mit Dopaminagonisten auf. Am häufigsten kommt es zu gestörter Impulskontrolle bezüglich

- Glücksspiel,

- Sexualtrieb,

- Essverhalten,

- Einkaufen,

- Internetaktivität.

Risikofaktoren sind:

- männliches Geschlecht,

- junges Alter,

- früher Krankheitsbeginn,

- Substanzmissbrauch,

- bipolare Erkrankungen,

- Spielsucht in der Eigen- oder Familienanamnese. Andere Störung der Impulskontrolle umfassen Punding (stereotypes Wiederholen sinnloser Tätigkeiten) und das Dopa-Dysregulationssyndrom (selbstständige, unkontrollierte Einnahme inadäquat hoher Dosen L-Dopa). Während die Impulskontrollstörung häufiger unter einer Therapie mit Dopaminagonisten beobachtet wird, kommt das Dopa-Dysregulationssyndrom insbesondere bei kurzwirksamen, hochpotenten dopaminergen Substanzen vor (Apomorphin, L-Dopa) [11].

Tipps

- In Hinblick auf die mitunter sehr schwerwiegenden sozialen und wirtschaftlichen Konsequenzen der Impulskontrollstörungen muss das Bewusstsein der Patienten, Angehörigen und Behandler für diese Nebenwirkung weiter gestärkt werden.

- Bei Hinweisen auf eine Impulskontrollstörung ist eine medikamentöse Umstellung notwendig.
Bei jüngeren Patienten sollte aufgrund der zu erwartenden langen Lebenserwartung mit einem Dopaminagonisten begonnen werden, um das Auftreten von Dyskinesien hinauszuzögern. Die Wirksamkeit der verschiedenen Non-Ergot-Dopaminagonisten auf die motorischen Symptome ist vergleichbar, aber insgesamt weniger effektiv als die von L-Dopa [10]. Bei der Auswahl des Präparates sollten die vom Patienten bevorzugte Applikationsform (Pflaster versus Tablette), seine Compliance bei der Tabletteneinnahme (retardiert versus unretardiert) und seine nichtmotorischen Symptome bedacht werden:

- Rotigotin wirkt sich besonders positiv auf Schlafstörungen aus.

- Pramipexol hat einen antidepressiven Effekt.

- Piribedil scheint weniger häufig zu Tagesmüdigkeit und Einschlafattacken zu führen als die übrigen Dopaminagonisten.

Wenn der Patient den erstgewählten Dopaminagonisten verträgt, sollte dieser bis zu einer effektiven Dosis titriert werden. Bei Auftreten von Nebenwirkungen (s. a. Infobox Impulskontrollstörung) ist ein Wechsel auf einen anderen Dopaminagonisten durchaus sinnvoll, da Metaanalysen darauf hinweisen, dass sich die Nebenwirkungsprofile unterscheiden können.

Bei älteren Patienten ist aufgrund von Komorbiditäten, häufigeren Einschränkung der kognitiven Leistungsfähigkeit und der Blutdruckregulation L-Dopa der Vorzug gegenüber Dopaminagonisten in der Primärtherapie zu geben.

Anticholinergika sind keine Medikamente der 1.Wahl beim IPS. Sie sollten nur bei anders nicht beherrschbarem Tremor bei jüngeren, kognitiv intakten Patienten unter Berücksichtigung des Nebenwirkungsprofils zur Anwendung kommen.

Nahrungsergänzungsmittel sind weiterhin sehr beliebt bei Patienten, da sie einfach verfügbar und meist gut verträglich sind. Für Coenzym Q10, Kreatin und ähnliche Substanzen ist allerdings kein klinischer Nutzen durch Studien belegt.

\section{Therapie der motorischen Komplikationen}

In der ELLDOPA-Studie wurde erstmals die dosisabhängige Wirkung sowie die Entwicklung von Dyskinesien von L-Dopa belegt. Bezüglich eines protektiven oder gar toxischen Effekts von L-Dopa auf die dopaminergen Neurone war die Studie allerdings nicht eindeutig [12]. Epidemiologische Studien weisen inzwischen darauf hin, dass ein verzögerter Beginn einer L-Dopa-Therapie das Auftreten von Dyskinesien nicht zu verzögern scheint. Dyskinesien treten unter L-Dopa-Therapie nach 4-6 Jahren bei mindestens der Hälfte und im weiteren Verlauf bei nahezu allen Patienten auf. 
Merke

Allgemein sollte daher bei Therapie mit L-Dopa eine möglichst niedrige, aber ausreichend wirksame Dosis gewählt werden.

Bei Auftreten von Dyskinesien können Dopaminagonisten, Amantadin und Safinamid als Add-ons verwendet werden. Daneben kommt es fast immer auch zu Wirkfluktuationen mit Wearing-off und mitunter schmerzhaften Off-Dystonien. Zur Minderung der Off-Phasen kommen zusätzlich Rasagilin und COMT-Hemmer zum Einsatz.

\section{Neue Therapieoptionen}

\section{FALLBEISPIEL \\ Beginnende Fluktuationen}

5 Jahre später kommt der gleiche Patient nach längerer Pause wieder in die Sprechstunde. Es sei ihm in den letzten Jahren gut gegangen, und er habe sich mit der Krankheit arrangiert. Im Verlauf habe die Monotherapie mit Ropinirol nicht mehr ausgereicht, sodass man L-Dopa hinzugenommen habe. Seit ca. einem halben Jahr falle es ihm nun aber schwerer, morgens aus dem Bett aufzustehen. Seine Frau bemerke, dass er manchmal seltsame Überbewegungen der Arme mache. Andererseits käme es tagsüber aber auch immer mal zum Nachlassen der Wirkung von L-Dopa. Die Dosis habe er selbstständig bereits angehoben; dennoch spüre er oft das Verlangen, mehr davon einzunehmen.

Der Patient führt für eine Woche ein ausführliches Bewegungsprotokoll. Bei der Wiedervorstellung wird hieraus ersichtlich, dass es jeweils eine Stunde nach der Einnahme von L-Dopa zu Dyskinesien und ca. drei Stunden später zu einem Wearing off kommt. Zur Verlängerung des Wirkintervalls wird Opicapon begonnen. Die Wirkung der Medikamente sei daraufhin in der Folge kontinuierlicher. Allerdings sei er vermehrt überbeweglich, was ihm in der sozialen Interaktion vor allem bei der Arbeit unangenehm sei. In den nächsten Monaten wird deshalb Safinamid ergänzt.

In den letzten Jahren sind in Deutschland zwei neue Medikamente zur Behandlung des IPS zugelassen worden:

Opicapon, seit Oktober 2016 in Deutschland verfügbar, ist neben Entacapon und Tolcapon der dritte erhältliche COMT-Hemmer, der als Add-on zu L-Dopa eingesetzt werden kann. Opicapon konnte in der Dosierung von $50 \mathrm{mg}$ in einem RCT die Off-Phasen um knapp 2 Stunden pro Tag reduzieren. Damit ist die Wirksamkeit von Opicapon in niedrigen Dosen mit der von 200 mg Entacapon vergleichbar. Vorteil im Vergleich zu Entacapon ist allerdings die nur einmal tägliche Gabe. Zudem kann unter Opicapon oft die L-Dopa-Dosis reduziert werden [13].

Safinamid ist seit Frühling 2015 als Add-on zu L-Dopa beim IPS mit Wirkfluktuationen und Dyskinesien in Deutschland zugelassen. Es bietet einen dualen Wirkmechanismus: Neben einer Hemmung der MAO-B wirkt es als Dopamin- und Glutamatmodulator, indem es aktivitätsabhängig den präsynaptischen Natriumeinstrom in glutamatergen Neuronen blockiert. Man geht davon aus, dass eine überschießende Glutamatfreisetzung zur Entstehung von Dyskinesien beiträgt.

Die Einnahme von Safinamid führte in der Zulassungsstudie über 24 Wochen zu einer durchschnittlichen Zunahme der Zeit im On um 1,5 Stunden pro Tag. Im Vergleich zu Placebo besserten sich UPDRS und Lebensqualität signifikant. Der Effekt blieb auch in einer Verlängerungsstudie über 2 Jahre nachweisbar. Die zugelassenen Dosierungen $50 \mathrm{mg}$ und $100 \mathrm{mg}$ unterschieden sich in der Wirksamkeit auf die Motorik kaum. Da in der Studie aber die höhere Dosierung stärkere Auswirkung auf die Lebensqualität hatte, sollte im klinischen Alltag eine Dosis von $100 \mathrm{mg}$ einmal täglich angestrebt werden [14].

\section{Kontinuierliche Therapie des fortgeschrittenen IPS}

\section{Tiefe Hirnstimulation}

\section{FALLBEISPIEL \\ Tiefe Hirnstimulation}

Zwei Jahre später berichtet der Patient, dass die Wirkfluktuationen weiter zugenommen hätten. Manchmal könne er inzwischen nicht mehr gut vorhersagen, ob, wann und wie die Medikamente wirken. Inzwischen sei er auch berentet worden. In der Indikationsprüfung für eine tiefe Hirnstimulation ergeben sich keine relevanten kognitiven Defizite, keine Depression und ein weiterhin sehr gutes Ansprechen im L-Dopa-Test. Nach ausführlicher Diskussion einigt man sich auf die Implantation einer tiefen Hirnstimulation im Nucleus subthalamicus. Drei Monate später kann die Dosis der dopaminergen Medikation auf ungefähr die Hälfte reduziert werden. Die Beweglichkeit sei deutlich gleichmäßiger im Tagesverlauf, auch nachts sei er gehfähig. Dass nun wieder regelmäßige Spaziergänge mit seiner Frau möglich sind, habe beiden Lebensqualität zurückgegeben. Aufgrund einer zunehmenden Dysarthrie werden die Stimulationsparameter noch einmal angepasst.

Die tiefe Hirnstimulation (THS, englisch Deep Brain Stimulation, DBS) stellt eine sehr effektive Therapie für ausgewählte Patienten mit medikamentös schwer 
beherrschbaren Fluktuationen und Dyskinesien oder einem medikamentös nicht kontrollierbaren Tremor dar. Die Indikation sollte allerdings in einem multidisziplinären Team sehr sorgfältig geprüft werden.

Die Evaluation der THS beinhaltet folgende Untersuchungen:

- eine kranielle MRT-Bildgebung,

- ein kurzes internistisches Work-up (EKG, Röntgenthorax und Abdomensonografie),

- eine logopädische, psychiatrische und ausführliche neuropsychologische Beurteilung.

- Außerdem sollte in einem standardisierten Dopa-Test die Wirksamkeit auf die motorischen Symptome und damit das Potenzial der THS festgestellt werden.

\section{PRAXISTIPP}

Als Faustregel ist festzuhalten, dass all die Symptome, die sich durch Dopa bessern, auch durch die THS im Nucleus subthalamicus (STN) positiv beeinflusst werden. Die Ausnahme stellt hier ein pharmakoresistenter Tremor dar, der durch die THS meist sehr effektiv behandelt werden kann.

Im Umkehrschluss gibt es auch typische Symptome, die durch die THS nicht behandelbar sind und teilweise sogar negativ beeinflusst werden können: Hierbei seien genannt: u. a. Freezing of Gait im medikamentösen On, Dysarthrie, kognitive Defizite und neuropsychiatrische Symptome.

Über diese Möglichkeiten und Grenzen der THS müssen Patienten ausführlich aufgeklärt werden, um eine unrealistische Erwartungshaltung zu vermeiden. Bei der Abschätzung von Therapiezielen sollten die individuellen Risiken und Limitationen des Patienten einbezogen werden.

Die EARLYSTIM-Studie verglich STN-THS mit bestmöglicher pharmakologischer Therapie in einem Patientenkollektiv, das deutlich jünger war und eine kürzere Krankheitsdauer und geringere L-Dopa-Äquivalenzdosis (LED) aufwies als die Kohorten vorheriger Studien. Hierbei wurde in der THS-Gruppe eine Überlegenheit gegenüber medikamentöser Therapie in Lebensqualität, motorischer Symptomschwere, Wirkfluktuationen und Aktivitäten des täglichen Lebens nachgewiesen. Komplikationen im Zusammenhang mit dem chirurgischen Vorgehen traten in $18 \%$ der Fälle auf [15].

\section{Merke}

Als Ergebnis der EARLYSTIM-Studie kann eine THS bei Patienten unter 60 Jahren auch in den ersten drei Jahren nach Diagnosestellung mit beginnenden Wirkfluktuationen empfohlen werden.
Bei diesen Patienten sollte allerdings nach Empfehlung der DGN-Leitlinie im Dopa-Test eine Verbesserung um mindestens $50 \%$ im UPDRS erreicht werden.

Bei Kontraindikationen für eine tiefe Hirnstimulation im subthalamischen Nukleus (STN) kommen bei einigen Patienten zwei alternative Zielpunkte in Betracht: der Globus pallidus internus (GPi) sowie Kerngebiete des Thalamus (ventraler intermedialer Nukleus, Vim).

Eine GPi-Stimulation führt zu einer ähnlichen Wirkung auf die motorischen Symptome wie eine STN-THS, allerdings ohne dass die dopaminerge Medikation signifikant reduziert werden kann. Neuropsychiatrische Nebenwirkungen treten seltener auf als bei einer STN-Stimulation. Die tiefe Hirnstimulation im Vim bleibt Patienten mit einem ganz im Vordergrund stehenden Tremor vorbehalten. Hierbei ist keine Besserung der übrigen motorischen Symptome zu erwarten.

Die seit wenigen Jahren verfügbare neue Generation von THS-Elektroden erlauben durch direktionale Kontakte eine feinere individuelle Anpassung des elektrischen Feldes der THS. Wie inzwischen in Studien nachgewiesen, lässt sich durch das neue Elektrodendesign ein größeres therapeutisches Fenster erreichen. Dies kann insbesondere bei suboptimaler Lage der Elektroden mit Vorliegen von stimulationsassoziierten Nebenwirkungen hilfreich sein.

Daneben finden sich in der Literatur immer mehr Hinweise auf den potenziellen Nutzen verschiedener spezieller Stimulationstechniken bei schwer kontrollierbaren Symptomen wie Freezing of Gait oder stimulationsinduzierter Dysarthrie, z. B. Interleaving-Stimulation, Änderung der Stimulationsfrequenz oder Impulsbreite. Hier bleiben aktuell noch die Ergebnisse größerer, verblindeter Studien abzuwarten.

\section{Infusionstherapien}

Eine effektive Alternative zur THS stellt die kontinuierliche Applikation von Medikamenten über Pumpen dar [16]. Hierfür stehen in Deutschland Apomorphin und Duodopa zur Verfügung. Beide Systeme werden meist tagsüber während der gesamten wachen Phase getragen und nur während der Nachtruhe ausgeschaltet.

Apomorphin ist ein schnell und kurz wirksamer Dopaminagonist mit einer sehr geringen oralen Bioverfügbarkeit, sodass er über eine subkutane Gabe zugeführt wird. Als Pen dient Apomorphin der schnellen Behandlung von abrupten Off-Phasen, als s. c. Pumpe der Minderung von Wirkfluktuationen. 
Apomorphin weist ein ähnliches Nebenwirkungsspektrum wie die übrigen Dopaminagonisten auf. Zusätzlich kann es zu Hautreizungen und Knötchenbildung an den Einstichstellen kommen. Der oft starken Übelkeit zu Beginn der Behandlung sollte durch medikamentöse Prophylaxe vorgebeugt werden. Jedoch können arterielle Hypotonie oder die Induktion von Halluzinationen oder Verwirrtheit bei einigen Patienten limitierende Nebenwirkungen seien.

Daneben bietet L-Dopa-Carbidopa Intestinal Gel (LCIG) oder Duodopa eine kontinuierliche Gabe von L-Dopa über einer PEG-/PEJ-Sonde. So wird eine schnellere Absorption bei vergleichbarer Bioverfügbarkeit und geringeren Schwankungen der L-DopaKonzentrationen im Tagesverlauf erreicht. Im Langzeitverlauf brechen allerdings ca. ein Fünftel der Patienten die Therapie ab. Neben fehlender Compliance oder Wirksamkeit sind besonders Komplikationen in Form von Sondendislokationen und -infektionen mögliche Gründe.

Als Entscheidungshilfe zur Pumpentherapie und zur tiefe Hirnstimulation mag $\triangleright$ Tab. 4 dienen [2].

\section{Nichtmotorische Symptome}

- Tab. 5 gibt einen Überblick über die wichtigsten nichtmotorischen Symptome und mögliche Behandlungsstrategien.

\section{Das fortgeschrittene IPS}

\section{Kognitive Störung}

\section{FALLBEISPIEL}

\section{Posturale Instabilität und Parkinson-Demenz}

Einige Jahre später kommt der Patient in Begleitung seiner Ehefrau zur Verlaufskontrolle. Leider habe seine kognitive Leistungsfähigkeit inzwischen deutlich nachgelassen, sodass er immer mehr Unterstützung seiner Frau im Alltag brauche. Nachts sei er oft unruhig, weil er ängstigende Halluzinationen habe. Trotz Umstellungen der Stimulationsparameter der THS stürze er weiterhin oft, meistens in Freezing-Episoden. Die Ehefrau empfindet inn in diesen Situationen oft als sehr ungestüm und unvorsichtig. Außerdem sei er aufgrund der Dysarthrie manchmal schwer verständlich, was ihn dann schnell verärgere. Insgesamt sei die ganze Familie belastet von der Situation.

Der Dopaminagonist wird aufgrund der Halluzinationen ausgeschlichen und Quetiapin zur Nacht hinzugenommen, wodurch der Patient deutlich ruhiger wird. Durch Gabe von Rivastigmin nimmt in der Folge auch die Sturzhäufigkeit etwas ab. Neben der regelmäßigen Physiotherapie werden Ergotherapie und Logopädie wieder aufgenommen. Hier liegt der Fokus nun auf dem Erhalt der Alltagsfunktionen.

-Tab. 4 Entscheidungshilfe Pumpentherapie und tiefe Hirnstimulation (modifiziert nach DGN-Leitlinie [2]).

\begin{tabular}{|c|c|c|c|c|c|}
\hline Kriterium & $\begin{array}{l}\text { Apomorphin s. } \\
\text { c. Pumpe }\end{array}$ & $\begin{array}{l}\text { L-Dopa-Pumpe } \\
\text { über PEJ }\end{array}$ & $\begin{array}{l}\text { tiefe Hirnstimu- } \\
\text { lation im STN }\end{array}$ & $\begin{array}{l}\text { tiefe Hirnstimu- } \\
\text { lation im GPi }\end{array}$ & $\begin{array}{l}\text { tiefe Hirnstimu- } \\
\text { lation im Thala- } \\
\text { mus (Vim) }\end{array}$ \\
\hline Alter $<70$ Jahre & ++ & ++ & ++ & ++ & ++ \\
\hline Alter $>70$ Jahre & + & ++ & - & + & + \\
\hline $\begin{array}{l}\text { leichtere kognitive } \\
\text { Störung }\end{array}$ & + & ++ & -1 & + & + \\
\hline PD-Demenz & - & + & - & - & + \\
\hline $\begin{array}{l}\text { medikamentös indu- } \\
\text { zierte Psychose }\end{array}$ & - & + & + & $(+)^{2}$ & + \\
\hline $\begin{array}{l}\text { therapieresistenter } \\
\text { Tremor }\end{array}$ & - & - & ++ & + & $++^{3}$ \\
\hline Testbarkeit & ++ & $+^{4}$ & - & - & - \\
\hline $\begin{array}{l}\text { eingeschränkte Ver- } \\
\text { sorgungs-/ } \\
\text { Pflegesituation }\end{array}$ & - & - & $(+)$ & $(+)$ & $(+)$ \\
\hline $\begin{array}{l}\text { + geeignet; ++ beson } \\
\text { GPi = Globus pallidu } \\
{ }^{1} \text { Häufiger neuropsycl } \\
{ }^{2} \text { Keine Medikamente } \\
{ }^{3} \text { Ausschließlich Wirkı } \\
{ }^{4} \text { Testphase über nas }\end{array}$ & $\begin{array}{l}\text { eeignet; - nicht g } \\
\text {-nus; STN = subtha } \\
\text { che Nebenwirkuns } \\
\text { ktion zu erwarten } \\
\text { if Tremor, nicht au } \\
\text { le Sonde vor der }\end{array}$ & $\begin{array}{l}\text { Is bei den anderer } \\
\text { übrigen motoriscl } \\
\text { PEJ-Anlage. }\end{array}$ & $\begin{array}{l}\text { entraler intermediale } \\
\text { nulationszielen. } \\
\text { ymptome! }\end{array}$ & ukleus & \\
\hline
\end{tabular}


Tab. 5 Häufige nichtmotorische Symptome und ihre Behandlung.

\begin{tabular}{|c|c|c|}
\hline Symptom & nichtmedikamentöse Therapie (Auswahl) & medikamentöse Therapie (Auswahl) \\
\hline Obstipation & $\begin{array}{l}\text { regelmäßige Bewegung ausreichende Flüssigkeits- } \\
\text { und Ballaststoffzufuhr }\end{array}$ & Macrogol Flohsamenschalen Prucaloprid \\
\hline Blasenentleerungsstörung & $\begin{array}{l}\text { Ausschluss bzw. Behandlung anderer Ursachen, } \\
\text { z. B. Prostatahyperplasie oder Beckenbodensen- } \\
\text { kung bei Nykturie: abendliche Koffeinkarenz }\end{array}$ & Anticholinergika \\
\hline erektile Dysfunktion & - & $\begin{array}{l}\text { Sildenafil (Cave bei orthostatischer } \\
\text { Hypotension) }\end{array}$ \\
\hline orthostatische Hypotension & $\begin{array}{l}\text { langsamer Lagewechsel ausreichende Flüssigkeits- } \\
\text { zufuhr bis } 3 \text { I pro Tag salzreiche Kost, häufigere, } \\
\text { kleinere Mahlzeiten Kompressionsstrümpfe Hochla- } \\
\text { gerung des Oberkörpers während der Nachtruhe } \\
\text { ggf. Reduktion der Einzeldosis von L-Dopa, wenn } \\
\text { zeitlicher Zusammenhang }\end{array}$ & $\begin{array}{l}\text { Sympathomimetika (Cave bei arterieller } \\
\text { Hypertonie in Ruhe) Fludrocortison }\end{array}$ \\
\hline Tagesmüdigkeit & $\begin{array}{l}\text { Schlafhygiene Wechsel / Absetzen des Dopamina- } \\
\text { gonisten (vor allem bei Einschlafattacken) }\end{array}$ & Methylphenidat / Modafenil \\
\hline Durchschlafstörung & $\begin{array}{l}\text { Schlafhygiene (regelmäßiger Schlaf-wach-Rhyth- } \\
\text { mus, „Routinen“, keine Bildschirmarbeit vor dem } \\
\text { Zubettgehen usw.) }\end{array}$ & $\begin{array}{l}\text { Rotigotin oder retardiertes Ropinirol (bei } \\
\text { nächtlicher Akinesie) Zopiclon Melatonin? }\end{array}$ \\
\hline Depression & $\begin{array}{l}\text { psychosoziale Beratung Psychotherapie } \\
\text { Lichttherapie? }\end{array}$ & $\begin{array}{l}\text { zunächst: ausreichende dopaminerge The- } \\
\text { rapie trizyklische Antidepressiva SSRI, } \\
\text { Venlafaxin }\end{array}$ \\
\hline $\begin{array}{l}\text { Störung des REM- } \\
\text { Schlafverhaltens }\end{array}$ & - & $\begin{array}{l}\text { Benzodiazepine, 1. Wahl Clonazepam (bei } \\
\text { selbst- oder fremdverletzendem Verhalten } \\
\text { im Schlaf) }\end{array}$ \\
\hline kognitive Störung & $\begin{array}{l}\text { Erhalt der sozialen Kontakte, anregende Umge- } \\
\text { bung Ergotherapie neuropsychologisches Training }\end{array}$ & Rivastigmin Donepezil (Off-Label Use) \\
\hline
\end{tabular}

Die leichte kognitive Störung (mild cognitive Impairment, $\mathrm{MCl}$ ) betrifft etwa ein Drittel der IPS-Patienten innerhalb der ersten 5 Krankheitsjahre. Hierbei ist eine frontale Dysfunktion mit Problemen in den Bereichen Aufmerksamkeit und Exekutivfunktionen am häufigsten, ohne dass es zu relevanten Einschränkungen im täglichen Leben kommt (in Abgrenzung zur Parkinson-Demenz). Das $\mathrm{MCl}$ der Exekutivfunktionen scheint nicht generell mit einem erhöhten Risiko für die Entwicklung einer Parkinson-Demenz (s. Infobox „Hintergrundinformation“) assoziiert zu sein. Störungen des Gedächtnisses und der visuellkonstruktiven Fähigkeiten hingegen zeigen das Fortschreiten der kognitiven Störung zu einer Demenz an.

\section{Merke}

Das Montreal Cognitive Assessment (MoCA) erfasst die Störungen frontalbasierter kognitiver Domänen besonders ausführlich, weshalb er dem Mini Mental StatusTest als globaler Suchtest für die kognitive Störung des IPS im klinischen Alltag vorgezogen werden sollte.

\section{HINTERGRUNDINFORMATION \\ Parkinson-Demenz}

Die Parkinson-Demenz ist definiert als eine kognitive Störung im Rahmen des IPS, die schwer genug ist, um Einschränkungen und Hilfsbedürftigkeit bei den Verrichtungen des täglichen Lebens zu bewirken. Im Langzeitverlauf des IPS weist die PD-Demenz eine Prävalenz von bis zu $80 \%$ mit einer Konversionsrate von bis zu $10 \%$ pro Jahr auf. Die PD-Demenz führt zu erhöhter Morbidität und deutlich reduzierter Lebensqualität. [17]

\section{Behandlung}

Nichtmedikamentöse, neuropsychologische Therapieformen (kognitives Training, Psychoedukation, Entspannungstechniken, neuropsychotherapeutische Strategien) haben sich in Studien als hilfreich bei Problemen mit Aufmerksamkeit, Gedächtnis und Problemlösung bei Parkinson-MCl und -Demenz erwiesen. Für die medikamentöse 
Therapie ist Rivastigmin weiterhin die 1. Wahl; alternativ kann Donepezil zum Einsatz kommen.

\section{Halluzinationen und Psychose}

Im Langzeitverlauf des IPS können - meist in Folge fortschreitender kognitiver Einschränkungen - Verwirrtheit, Psychose und visuelle Halluzinationen auftreten, die durch die dopaminerge Therapie begünstigt werden.

\section{Merke}

Bei einer akuten Verschlechterung müssen immer zuerst Infekte, Exsikkose, metabolische Störungen und auslösende ZNS-wirksame Medikamente als Ursachen in Betracht gezogen und behandelt werden.

Wenn diese Maßnahmen zu keiner ausreichenden Besserung führen, sollte die Parkinson-Medikation reevaluiert und in folgender Reihenfolge reduziert sowie ggf. abgesetzt werden:
- Anticholinergika
- Amantadin
- Dopaminagonisten
- L-Dopa

Wenn auch die Reduktion der dopaminergen Medikation nicht ausreichend ist, kann als Clozapin zum Einsatz kommen. Es ist als einziges Neuroleptikum für Anwendung beim IPS empfohlen. Aufgrund des Risikos für eine Agranulozytose sind regelmäßige Blutbildkontrollen erforderlich, was die Gabe gerade bei Patienten mit reduzierter Mobilität oder Compliance erschweren kann. Aus den Reihen der Neuroleptika stellt Quetiapin aktuell die einzige Alternative dar - mit allerdings deutlich geringerem antipsychotischem Potenzial.

Mit Pimavanserin ist in den USA das erste Medikament speziell für die Behandlung von Halluzinationen und Psychose bei IPS zugelassen. Pimavanserin entfaltet seine Wirkung an verschiedenen Subtypen des Serotoninrezeptors. Dagegen hat es keine Affinität zum Dopaminrezeptor und soll daher zu keiner Verschlechterung der motorischen Symptome führen. In der Zulassungsstudie war Pimavanserin effektiv in der Behandlung von Illusionen und Halluzinationen bei $80 \%$ der Patienten, verglichen zu 58\% in der Placebogruppe. [18] Damit ist die antipsychotische Wirkung zwar geringer als die von Clozapin; es traten aber auch weniger Nebenwirkungen auf. Pimavanserin ist bisher in der EU und Deutschland nicht zugelassen.

\section{Posturale Instabilität und Stürze}

Im Verlauf der Erkrankung kommt es zu einer zunehmenden posturalen Instabilität, deren Folgen eingeschränkte Mobilität, Angst, sozialer Rückzug und Stürze und damit verbundene Komplikationen sind.

\section{Medikamentöse Behandlung}

Rivastigmin kann neben der Wirkung auf die kognitive Störung auch einen positiven Einfluss auf psychotische Symptome und auf die Gangstörung bei Patienten mit regelmäßigen Stürzen haben, wie die RESOND-Studie gezeigt hat [19].

\section{Nichtmedikamentöse Behandlung}

Eine Vielzahl von Studien belegt den Nutzen von Physiotherapie auf die motorischen Symptome und das Gangbild von IPS-Patienten [20]. Physiotherapie scheint sich außerdem positiv auf Aspekte wie globale kognitive Funktion und einzelne spezifische kognitive Domänen (mentale Flexibilität, Aufmerksamkeit und Verarbeitungsgeschwindigkeit) auszuwirken [21].

Der Oberbegriff Physiotherapie umfasst ein weites Spektrum unterschiedlicher Formen der Bewegungstherapie. Patienten interessieren sich oft besonders für Empfehlungen bezüglich der Wirksamkeit spezieller Formen der Bewegung. Inzwischen existieren neben den Studien zu Tango u.a. auch einzelne Studien zu Paartanz, Irish Dance, Zumba, Samba, Yoga, Nordic Walking und Ballett. Einige Untersuchungen hatten durchaus ein positives Ergebnis auf motorische Funktionen oder Lebensqualität zu verzeichnen. Im Allgemeinen unterscheiden sich die Studien allerdings stark in Qualität, Stichprobengröße, Methodik, Interventions- und Nachbeobachtungszeitraum, was die Interpretation der Ergebnisse und die Ableitung allgemein gültiger Empfehlungen sehr schwierig macht.

TIPP

Dennoch sollten die positiven Aspekte regelmäßiger nichtmedikamentöser Therapie nicht außer Acht gelassen werden: Sie leistet einen elementaren Beitrag zur Verbesserung und zum Erhalt der Alltagskompetenzen, der Mobilität und der sozialen Integration der Patienten.

\section{Zusammenfassung}

Trotz aller wissenschaftlichen Aktivität in der Entwicklung neuroprotektiver Therapien ist das idiopathische Parkinson-Syndrom weiterhin eine unheilbare neurodegenerative Erkrankung. Somit sollten die bestmögliche symptomatische Behandlung und damit die Besserung der Lebensqualität der Patienten im Fokus unserer Aufmerksamkeit stehen. Wie in diesem Beitrag dargestellt, steht uns heute eine Vielzahl an effektiven medikamentösen und nichtmedikamentösen Optionen zur Verfügung, um Patienten in verschiedenen Krankheitsstadien eine individualisierte Therapie anzubieten. 


\section{KERNAUSSAGEN}

- Die Diagnose des idiopathischen Parkinson-Syndroms (IPS) beruht weiterhin auf einer ausführlichen Anamnese und neurologischen Untersuchung sowie dem Nachweis der DopaResponsivität.

- In klinisch nicht eindeutigen Fällen kann zur Differenzialdiagnostik nuklearmedizinische Diagnostik eingesetzt werden: das FP-CIT-SPECT zur Abgrenzung des IPS gegen nicht neurodegenerative Ursachen sowie die 18F-FluordesoxyglukosePositronenemissionstomografie (FDG-PET) zur Differenzierung gegenüber atypischen ParkinsonSyndromen.

- Die primäre Therapie richtet sich nach dem Alter des Patienten: Dopaminagonisten bei jüngeren Patienten und L-Dopa bei älteren. Daneben sollten aber immer auch die individuellen Besonderheiten des Patienten bei der Auswahl der Medikamente berücksichtigt werden.

- Mit dem Catechol-O-Methyltransferase-(COMT-) Hemmer Opicapon sowie Safinamid mit seinem dualen Wirkmechanismus stehen zwei neue Optionen für die medikamentöse Behandlung des IPS mit Wirkfluktuationen zur Verfügung.

- Die tiefe Hirnstimulation (THS) stellt eine sehr effektive Therapie des IPS auch bei Patienten mit beginnenden Fluktuationen dar, sofern die Indikationsprüfung von einem erfahrenen, multidisziplinären Team durchgeführt wird.

- Die kontinuierliche Applikation von L-Dopa oder Apomorphin bietet auch Patienten mit einem fortgeschrittenen IPS und Kontraindikationen für die tiefe Hirnstimulation eine effektive Therapie von Wirkfluktuationen.

- Kognitive Defizite, Stürze und psychotische Symptome sind typische Komplikationen des fortgeschrittenen IPS, denen aufgrund ihrer hohen Komplikationsraten besondere Aufmerksamkeit geschenkt werden sollte.

- Nichtmedikamentöse Therapien wie Physio- und Ergotherapie, Logopädie sowie kognitives Training sind entscheidend für den Erhalt der Alltagskompetenz, der Lebensqualität und der sozialen Integration und sollten präventiv schon vor Auftreten einer relevanten Beeinträchtigung eingesetzt werden.

\section{Interessenkonflikt}

Die Autoren geben an, dass keine Interessenkonflikte vorliegen.

\section{Autorinnen / Autoren}

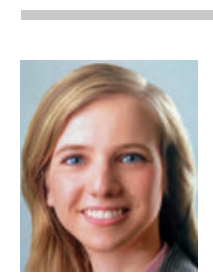

\section{Josefine Waldthaler}

Dr. med., Jahrgang 1988. 2007-2013 Studium der Humanmedizin an der Otto-von-Guericke Universität Magdeburg. 2013-2018 Facharztausbildung Neurologie. 2016-2017 Forschungsaufenthalt Institut für Klinische Neurowissenschaften, Karolinska Institut Stockholm. Seit 2017 Klinik für Neurologie am Universitätsklinikum Marburg. Schwerpunkt: Kognition und Augenbewegungen bei Bewegungsstörungen, tiefe Hirnstimulation.

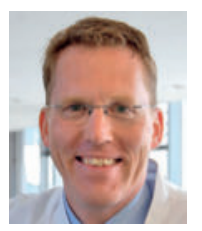

\section{Lars Timmermann}

Prof. Dr. med., Jahrgang 1972, 1992-1999 Studium der Humanmedizin an der ChristianAlbrechts-Universität zu Kiel. 2000-2005 Facharztausbildung Neurologie. 2008-2016 Professur Bewegungsstörungen Universität zu Köln. Seit 2016 W3-Professur und Direktor der Klinik für Neurologie am Universitätsklinikum Marburg. Schwerpunkt: Pathophysiologie von Bewegungsstörungen, tiefe Hirnstimulation.

\section{Korrespondenzadresse}

\section{Dr. med. Josefine Waldthaler}

Universitätsklinikum Gießen und Marburg, Standort Marburg Klinik für Neurologie

Baldingerstraße

35033 Marburg

E-Mail: josefine.waldthaler@staff.uni-marburg.de

\section{Wissenschaftlich verantwortlich gemäß}

\section{Zertifizierungsbestimmungen}

Wissenschaftlich verantwortlich gemäß Zertifizierungsbestimmungen für diesen Beitrag ist Dr. med. Josefine Waldthaler, Marburg.

\section{Erstveröffentlichung}

Dieser Beitrag wurde erstveröffentlicht in: Neurologie up2date 2019; 2: 31-46.

\section{Literatur}

[1] Postuma RB, Berg D, Stern M et al. MDS clinical diagnostic criteria for Parkinson's disease. Mov Disord 2015; 30: 15911601

[2] Deutsche Gesellschaft für Neurologie - DGN. S3-Leitlinie Idiopathisches Parkinson-Syndrom. In: Leitlinien für Diagnostik und Therapie in der Neurologie. DGN; 2016. Im Internet: https: / /www.awmf.org/leitlinien/detail/II/030-010.html; Stand: 28.12 .2018

[3] Danel SE, Lees AJ. Parkinson's Disease Society Brain Bank, London: overview and research. J Neural Transm Suppl 1993; 39: $165-172$ 
[4] Pavese N, Tai YF. Nigrosome imaging and neuromelanin sensitive mri in diagnostic evaluation of Parkinsonism. Mov Disord Clin Pract 2018; 5: 131-140

[5] Tripathi M, Dhawan V, Peng S et al. Differential diagnosis of parkinsonian syndromes using F-18 fluorodeoxyglucose positron emission tomography. Neuroradiology 2013; 55: 483-492

[6] Domingo A, Klein C. Genetics of Parkinson Disease. Handb Clin Neurol 2018; 147: 211-227. doi:10.1016/B978-0-44463233-3.00014-2

[7] Berg D, Postuma RB, Adler CH et al. MDS research criteria for prodromal Parkinson's disease. Mov Disord 2015; 30: 16001611

[8] Fereshtehnejad SM, Romenets SR, Anang JBM et al. New clinical subtypes of Parkinson disease and their longitudinal progression. JAMA Neurol 2015; 72: 863-873

[9] Seppi K, Weintraub D, Coelho $\mathrm{M}$ et al. The movement disorder society evidence-based medicine review update: Treatments for the non-motor symptoms of Parkinson's disease. Mov Disord 2011; 26 (Suppl 3): S42-S80

[10] Fox SH, Katzenschlager R, Lim SY et al. International Parkinson and movement disorder society evidence-based medicine review: Update on treatments for the motor symptoms of Parkinson 's disease. Mov Disord 2018; 33: 1248-1266

[11] Weintraub D, David AS, Evans AH et al. Clinical spectrum of impulse control disorders in Parkinson's disease. Mov Disord 2015; 30: 121-127

[12] Fahn S, Parkinson Study Group. Does levodopa slow or hasten the rate of progression of Parkinson's disease? J Neurol 2005; 252 (Suppl 4): IV37-IV42

[13] Ferreira JJ, Lees A, Rocha JF et al. Opicapone as an adjunct to levodopa in patients with Parkinson's disease and end-ofdose motor fluctuations: A randomised, double-blind, controlled trial. Lancet Neurol 2016; 15: 154-165

[14] Schapira AHV, Fox SH, Hauser RA et al. Assessment of safety and efficacy of safinamide as a levodopa adjunct in patients with Parkinson disease and motor fluctuations: a randomized clinical trial. JAMA Neurol 2017; 74: 216-224

[15] [No authors listed]. Deep brain stimulation for Parkinson's disease with early motor complications. JAMA 2014; 311: 1686-1687

[16] Antonini A, Moro E, Godeiro C et al. Medical and surgical management of advanced Parkinson's disease. Mov Disord 2018; 33: 900-908

[17] Vasconcellos LFR, Pereira JS. Parkinson's disease dementia: Diagnostic criteria and risk factor review. J Clin Exp Neuropsychol 2015; 37: 988-993

[18] Mathis M V, Muoio BM, Andreason P et al. The US Food and Drug Administration's perspective on the new antipsychotic pimavanserin. J Clin Psychiat 2017; 78: 668-673

[19] Henderson E], Lord SR, Brodie MA et al. Rivastigmine for gait stability in patients with Parkinson's disease (ReSPonD): a randomised, double-blind, placebo-controlled, phase 2 trial. Lancet Neurol 2016; 15: 249-258

[20] Tomlinson CL, Patel S, Meek C et al. Physiotherapy versus placebo or no intervention in Parkinson's disease. Cochrane Database Syst Rev 2013; (9): CD002817. doi:10.1002/ 14651858.CD002817.pub4

[21] Da Silva FC, lop RDR, De Oliveira LC et al. Effects of physical exercise programs on cognitive function in Parkinson's disease patients: A systematic review of randomized controlled trials of the last 10 years. PLoS One 2018; 13: e0193113. doi:10.1371/journal.pone. 0193113

Bibliografie

DOI https://doi.org/10.1055/a-0952-8075

Fortschr Neurol Psychiatr 2019; 87: 445-461

(c) Georg Thieme Verlag KG Stuttgart · New York ISSN 0720-4299 


\section{Punkte sammeln auf CME.thieme.de}

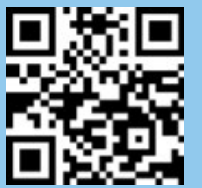

Diese Fortbildungseinheit ist in der Regel 12 Monate online für die Teilnahme verfügbar. Den genauen Einsendeschluss finden Sie unter https://eref.thieme.de/CXDEGBL.

Sollten Sie Fragen zur Online-Teilnahme haben, finden Sie unter https://cme.thieme.de/hilfe eine ausführliche Anleitung. Wir wünschen viel Erfolg beim Beantworten der Fragen!

Unter https://eref.thieme.de/CXDEGBL oder über den QR-Code kommen Sie direkt zur Startseite des Wissenstests.

VNR 2760512019156642041

\section{Frage 1}

Welche Aussage zur Diagnostik des idiopathischen Parkinson-Syndroms (IPS) ist richtig?

A Mit den UK-Brain-Bank-Kriterien allein lässt sich die klinische Diagnose mit größter Genauigkeit stellen.

B Das IPS grenzt sich gegenüber anderen, atypischen Parkinson-Syndromen durch Auffälligkeiten in der Magnetresonanztomografie ab („Kolibri“-Zeichen).

C Der Dopa-Test ist gerade bei De-Novo-Patienten für eine Differenzierung zwischen IPS und atypischen Parkinson-Syndromen geeignet.

D Die Bradykinese ist das wegweisende Symptom für die Diagnose eines IPS.

E Die Diagnose eines klinisch etablierten IPS ist zu stellen, wenn zusätzlich zum Hauptkriterium ein unterstützendes Merkmal vorliegt.

\section{Frage 2}

Wann sollte zur Überprüfung von Diagnose und Therapieerfolg das erste Follow-up des Patienten erfolgen?
A nach 14 Tagen
B nach 1 Monat
C nach 3 Monaten
D nach 6 Monaten
E nach 1 Jahr

\section{Frage 3}

Welche der folgenden Substanzen sollte nicht für die primäre Monotherapie des frühen idiopathischen Parkinson-Syndroms eingesetzt werden?
A L-Dopa
B Entacapon
C Rasagilin
D Ropinirol
E Pramipexol

\section{Frage 4}

Welche Aussage zum Auftreten von Dyskinesien ist falsch?

A Bei langjähriger L-Dopa-Therapie ist innerhalb von 4-6 Jahren bei ca. 50 \% der Behandelten mit Dyskinesien zu rechnen.
B Ältere Patienten sollten aufgrund von z. B. Komorbiditäten bevorzugt mit L-Dopa behandelt werden, auch wenn ein Dyskinesie-Risiko besteht.

C Bei jüngeren Patienten empfiehlt sich ein Therapiebeginn mit Dopaminagonisten, um Dyskinesien hinaus zu zögern.

D Eine tiefe Hirnstimulation kommt nach interdisziplinärer Prüfung bei medikamentös schwer beherrschbaren Dyskinesien infrage.

E Dyskinesien lassen sich durch Anticholinergika gut kontrollieren.

\section{Frage 5}

Welche Aussage zur Parkinson-Demenz stimmt?

A Bei der Parkinson-Demenz handelt es sich um eine medikamentös induzierte, reversible kognitive Störung.

B Die Parkinson-Demenz führt zu relevanten Einschränkungen im Alltag des Patienten.

C Die Diagnose einer Parkinson-Demenz sollte immer über den Mini Mental Status-Test erfolgen.

D Neuropsychologische Therapien wie kognitives Training, Psychoedukation etc. sind bei Parkinson-Demenz ineffektiv.

E Gegen die Symptome der Parkinson-Demenz sind noch keine Medikamente verfügbar.

\section{Frage 6}

Was trifft auf die Impulskontrollstörung beim idiopathischen Parkinson-Syndrom zu?

A Impulskontrollstörungen treten am häufigsten als Nebenwirkung von L-Dopa auf.

B Impulskontrollstörungen betreffen Frauen häufiger als Männer.

C Bei Hinweisen auf eine Impulskontrollstörung ist eine medikamentöse Umstellung notwendig.

D Impulskontrollstörungen sollten primär mit Verhaltenstherapie behandelt werden.

E Bei Patienten mit Impulskontrollstörungen besteht immer ein ausgeprägter Leidensdruck. 


\section{Punkte sammeln auf CME.thieme.de}

Fortsetzung ...

\section{Frage 7}

Welche medikamentöse Therapie ist bei dem jeweilig genannten nichtmotorischen Symptom des idiopathischen Parkinson-Syndroms kontraindiziert?

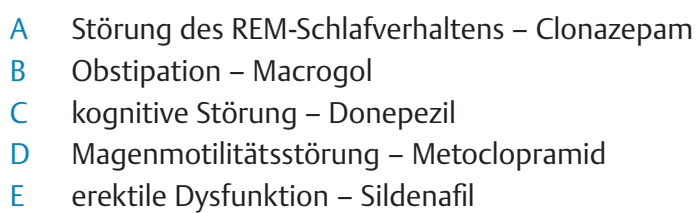

\section{Frage 8}

Einer der folgenden Befunde stellt keine klare Kontraindikation für die tiefe Hirnstimulation im Nucleus subthalamicus dar. Welcher?
A medikamentenresistenter Tremor
B Alter 78 Jahre
C L-Dopatest mit einer Verbesserung in der Unified Parkin- son's Disease Rating Scale (UPDRS) um 15\%
D deutlich eingeschränkte kognitive Fähigkeiten in der neuropsychologischen Testung
E behandlungsbedürftige schwere Depression

\section{Frage 9}

Welche Aussage zu Nebenwirkungen medikamentöser Therapien des idiopathischen Parkinson-Syndroms (IPS) ist falsch?

A Bei Gabe von Tolcapon regelmäßige Leberfunktionskontrolle veranlassen.

B Bei Non-Ergot-Dopaminagonisten sind eingeschränkte Fahrtauglichkeit und Impulskontrollstörungen möglich.
C Rasagilin und Selegilin wechselwirken mit verschiedenen anderen Wirkstoffen, wie z. B. SerotoninWiederaufnahmehemmern.

D Ergot-Dopaminagonisten können als Nebenwirkung Fibrosen verursachen und dürfen nur in Ausnahmefällen verordnet werden.

E Alle Parkinson-Medikamente sind nebenwirkungsarm und daher gleichermaßen für die Behandlung geeignet.

\section{Frage 10}

Welches der folgenden Medikamente sollte zur Behandlung der Psychose im Rahmen eines idiopathischen Parkinson-Syndroms eingesetzt werden?
A Risperidon
B Clozapin
C Olanzapin
D Promethazin
E Amisulprid 